\title{
Evidence for the dependence of the SARS-Cov-2 Delta high diffusivity on the associated N:G215C nucleocapsid mutation
}

\section{Vito Marchitelli}

Department of Mobility, Public Works, Ecology, Env, Puglia Region Government, 70100 Bari, Italy

\section{Claudia Troise}

INGV, Osservatorio Vesuviano, 80124 Naples, Italy

\section{Antonio Parisi}

Istituto Zooprofilattico Sperimentale della Puglia e della Basilicata, 70121 Foggia, Italy

\section{Angelica Bianco}

Istituto Zooprofilattico Sperimentale della Puglia e della Basilicata, 70121 Foggia, Italy

\section{Laura Del Sambro}

Istituto Zooprofilattico Sperimentale della Puglia e della Basilicata, 70121 Foggia, Italy

\section{Renato Somma}

INGV, Osservatorio Vesuviano, 80124 Naples, Italy

Antonio Coviello

5 CNR-IRISS, 80134 Naples, Italy

\section{Paolo Harabaglia}

Scuola di Ingegneria, Università della Basilicata, 85100, Potenza, Italy

\section{Felice Ungaro}

Department of Health, Puglia Region Government, 70121 Bari, Italy

Giuseppe De Natale ( $\sim$ giuseppe.denatale@ingv.it)

INGV, Osservatorio Vesuviano, 80124 Naples, Italy

\section{Research Article}

Keywords: COV-Sars-2 pandemics, Delta Variant of Concern, High Diffusivity, Nucleocapsid mutation

Posted Date: August 26th, 2021

DOI: https://doi.org/10.21203/rs.3.rs-846225/v1

License: (c) (1) This work is licensed under a Creative Commons Attribution 4.0 International License. Read Full License 


\section{Abstract}

The continuous evolution of the SARS-CoV-2 virus genome and the consequent substitutions observed in the amino acid sequences, can induce significant changes in parameters such as diffusivity and pathogenicity, and causes constant concern regarding the efficacy of vaccines against the new variants in circulation. In recent months there has been an increase in the number of infections first in India, and more recently in the United Kingdom. The genome sequencing of the samples showed that this increase coincided with the emergence of a new variant, B.1.617.2, also known as VOC Delta. This variant is rapidly becoming dominant in several countries, causing increasing concern for its extreme diffusivity and its ability to often overcome the vaccines.

The high diffusivity of this variant is normally ascribed to the Spike protein mutations. However, we will show here that it is rather due to the nucleocapsid substitution N:G215C. This is made clear by comparing, in the genomic sequences available on the GISAID database, the relative increase of the Delta variant with and without the associated N:G215C substitution. Once the extreme diffusivity of the Delta variant with associated nucleocapsid aminoacidic substitution is evidenced, we tentatively explain it as possibly due to the adaptive effect of the highly selective environment in Countries with high levels of vaccination.

\section{Introduction}

Since the end of 2019, SARS-CoV-2 has spread all over the World, causing a serious disease called COVID-19, responsible, till now, for over 4.3 millions of deaths. After the first appearance in the city of Wuhan, China, the original strain has genetically changed several times, generating 'Variants of Concern' (VOC), which have been highlighted and strictly monitored for their higher diffusivity and/or their increased harmfulness (Boehm et al., 2021). The first Western Country to be strongly hit by the virus has been Italy, experiencing one of the highest levels of mortality (e.g. De Natale et al., 2020a). The last, and most concerning VOC is the so called SARS-CoV-2 'Delta' variant, also known as lineage B.1.617.2. It was firstly detected in India in late 2020, and the WHO named it 'Delta' on 31 May 2021 (ECDC, 2021). The Delta variant is actually thought to be extremely diffusive, since it has become dominant among sequenced viral genomes in many Countries all over the World (ECDC, 2021). It also determined peaks of infections in several Countries that, after massive vaccination campaigns, were experiencing very low incidence levels (Mattiuzzi et al., 2021).

It harbors nucloetidic variations in the gene encoding the SARS-CoV-2 spike protein (Shang et al., 2020) causing the aminoacidic substitutions T478K, P681R and L452R (Starr et al., 2021) which are supposed to affect transmissibility of the virus as well as a lower affinity for the neutralizing antibodies for previously circulating variants of the COVID-19 virus (ECDC, 2021).

Till now, attention has been mainly paid to aminoacidic variations of the Spike gene of the virus, but some important aminoacidic variations affecting the Nucleocapside gene of the virus, together with 
corresponding antibodies, have been described (Brochot et al., 2020; Burbelo et al., 2020; Liang et al., 2021; Wu et al., 2021; Mourier et al., 2021).

The immunological mechanisms concerning the nucleocapsid and the possible effects that the changes of this protein can have on transmissibility, replicability and pathogenicity of the virus are still mostly unclear. Recently, evidence has been found supporting the role played by certain nucleocapsid aminoacidic substitutions in promoting higher diffusivity, infectivity and virulence of SARS-CoV-2 (Wu et al., 2021).

In the present study, based on accurate observations of genetic sequences of SARS-CoV-2 deposited in GISAID database (https://www.gisaid.org/) we analyse the evolution over time of the Delta variant with wild type aminoacid N:215G, as compared to the variants 'Alpha', and to the Delta variant associated to the Nucleocapsid aminoacidic substitution N:G215C. Such a nucleocapsid mutation has never been considered in literature till now, although we will show its evolution is worthy of note. This comparison hence shows results of extreme interest, which someway upsets what is normally assumed today, and could have profound implications for the containment strategies.

\section{Data Analysis And Results}

All the data and analyses shown in this work have been taken from the GISAID database containing the genomes of SARS-CoV-2 sequenced worldwide (https://www.gisaid.org/).

The relative incidences of the Alpha and Delta variants genomes, starting from February 13th until July 31 st 2021 and available in the GISAID website, were plotted. The plot for UK and worldwide data are shown in Fig. 1 and Fig. 2, respectively. The two figures also show the daily number of new infections recorded in the indicated periods.

From Fig. 1, it is very clear that, since middle February, the daily number of new infections starts to decline, while the Alpha variant is still dominant. The minimum of daily new infections coincides with a period, from the beginning of April till the end of May, in which the Alpha variant starts to decline, and the Delta variant starts to rapidly increase. In the middle of June, the Alpha variant is almost zeroed, whereas the Delta variant already represents more than $90 \%$ of the sequenced genomes. After the first week of June, the daily number of new infections starts to significantly increase, reaching a peak in middle July. Such a significant increase in the daily infection number clearly correlates with the Delta variant having become dominant; it suggests this variant is more contagious, as previously described (ECDC 2021). However, in the figures we separated the genomes containing the Delta variant in two groups: with and without the substitution N:G215C. As it is clear from the Fig. 1, the incidence of Delta variant without the substitution starts to increase from the end of April, reaching a maximum of slightly more than $10 \%$ of the total genomes from the end of May, and then remains stable with a slight decay starting after the middle of June. On the contrary, what is really sharply increasing, becoming rapidly dominant, is the Delta variant containing the N:G215C substitution. Since the end of June, genomes classified as Delta variant containing the substitution N:G215C, reached about $95 \%$ of incidence in the sequenced genomes, 
whereas Alpha variant has become almost zeroed and the Delta variant N:215G represents almost all the remaining $5 \%$.

Figure 2 shows the incidence of the main variants in the GISAID database all over the World. The main features are very similar to what already noted for the UK catalogue (which anyway represents a large part of the sequenced genomes catalogue): it is evident the decline of the Alpha variant and the rise of the Delta ones, with the Delta associated to the nucleocapside substitution N:G215C rapidly increasing and becoming dominant with over $80 \%$ of the sequentiated genomes, while the Delta mutation, lacking the N:G215C substitution, represents around $10 \%$ or less of the total number. It should be noted, however, that the worldwide catalogue GISAID mostly reflects the situation of the most advanced Countries, i.e. Western Countries and China, which actually make the large majority of genome sequentiations.

\section{Discussion And Conclusion}

From the accurate analysis of the GISAID catalogue of sequenced SARS-CoV-2 genomes, it is evident the evolution of the main variants which have dominated the pandemic scenario infection in the last year. Alpha variant, which has been the dominant one for several months, started to decline in the UK since the middle of April, and worldwide since the middle of May (about one month later). The decline of the Alpha variant is accompanied by the rise of the Delta variant. However, the increase of the Delta variant alone is rather slow, and reach a maximum of about $10 \%$ of the whole sequenced genomes in a given time interval (it happens around the beginning of June in the UK, and one-two weeks later worldwide); what it is really fast is the increase of the Delta variant associated to the N:G215C substitution which rapidly reached (around the end of June in UK, and about one month later worldwide) a very large fraction of the total genomes (around $95 \%$ in UK, and more than $80 \%$ worldwide). In UK, the Delta variant, with or without the N:G215C substitution, actually represents close to $100 \%$ of the total of sequenced genomes, since the end of June; worldwide, the Delta variant represents about $90 \%$ of the total circulating genomes (with the warning that, since the most of the sequenced genomes were collected in UK, the worldwide catalogue is likely biased by an overestimation of the Delta variant in the catalogue, with respect to the actually circulating strains).

Besides the fundamental observation that is not the Spike variations alone, in the Delta variant, which makes it so diffusive and rapidly dominant, but rather the association with the nucleocapside substitution N:G215C, it remains to understand why it occurs.

Although this goal is out of the scope of the present study, there is growing evidence for the important role played by SARS-CoV-2 nucleocapsid substitutions for diffusivity, infectivity and virulence of the different strains.

Recently, other noteworthy mutations of the nucleocapsid of the SARS-CoV-2 have been detected and studied (Badua et al., 2021; Rahman et al., 2021). As an example, the mutation 203K/204R has been demonstrated to have competitive advantages as well as increased infectivity and virulence (Wu et al., 2021). 
The rapid growth and the almost complete dominance of this mutation in UK, as compared with the rest of World (see for example India, in the following discussion), could likely indicate a selective process due to the high vaccination rates in UK. In a highly competitive environment, in fact, like UK in which, already in May, a very high fraction of population was vaccinated, only the most fit mutations can spread, in a very selective way. This hypothesis agrees with the observation that the Delta variant with N:G215C nucleocapsid mutation is rapidly becoming dominant in all European countries and USA, as soon as high fractions of vaccinated people have been reached. The differential effect of diffusion of the two kinds of Delta variant, as related to different vaccination degrees, seems corroborated by comparing the evolution of the Alpha and Delta variants between UK and India, where Delta variant was firstly detected. In Fig. 3 the time evolution of Alpha variant, Delta variant without N:G215C and Delta variant with N:G215C substitution, in the two Countries. It is clear that in India, which is characterized by a much lower fraction of vaccinated people, the two kinds of Delta variant reach similar levels, both occupying a maximum around $50 \%$ of the total genomes. In the last months, not shown in the figure (times for India have been shifted so to put in phase with UK the rise of Delta variant: see caption of Fig. 3), the Delta N:G215C has reached in India about $65 \%$ of prevalence, with the Delta variant without the N:G215C occupying the remaining $35 \%$ of the sequentiated genomes in the Country.

The ability of Delta variant associated with this particular nucleocapsid mutation to spread also (and mainly) in populations with high vaccination rates should strongly warn us for the future management of the pandemic. In addition, all the observations about possible increase of virulence of this variant, including hospitalization and death, have been made during the Summer 2021, which is the starting period of its diffusion. Actually, several works pointed out the strong seasonality of COVID-19, with Summer months experiencing much less infection diffusion and milder disease (e.g. De Natale et al., 2020b; Nicastro et al., 2021).

Our observations confirms the need to accurately monitoring and studying the whole viral genome, in addition to the Spike mutations, with the aim to identify variations responsible for increasing of viral fitness.

\section{Declarations}

Competing Interests: The authors declare they have no competing interests.

\section{References}

Badua, C.L.D.C., Baldo, K.A.T. and Medina, P.M.B., 2021. Genomic and proteomic mutation landscapes of SARS-CoV-2. J. Med. Virol. 93, 1702-1721. doi:10.1002/jmv.26548.

Brochot, E., Demey, B., Touzé, A., Belouzard, S., Dubuisson, J., Schmit, J.L., Duverlie, G., Francois, C., Castelain, S. and Helle, F., 2020. Anti-spike, Anti-nucleocapsid and Neutralizing Antibodies in SARS-CoV-2 Inpatients and Asymptomatic Individuals. Front. Microbiol. 11:584251. doi: 10.3389/fmicb.2020.584251 
Burbelo, P.D., Riedo, F.X., Morishima, C. et al., 2020. Sensitivity in Detection of Antibodies to Nucleocapsid and Spike Proteins of Severe Acute Respiratory Syndrome Coronavirus 2 in Patients With Coronavirus Disease 2019. J. Infect. Dis. 222, 2, 206-213. doi:10.1093/infdis/jiaa273

Bobik, T.V., Kostin, N.N., Skryabin, G.A., Tsabai, P.N., Simonova, M.A., Knorre, V.D., Mokrushina, Y.A., Smirnov, I.V., Kosolapova, J.A., Vtorushina, V.V., Inviyaeva, E.V., Polushkina, E., Petrova, U.L., Levadnaya, A.V., Krechetova, L.V., Shmakov, R.G., Sukhikh, G.T., Gabibov, A.G. Epitope-Specific Response of Human Milk Immunoglobulins in COVID-19 Recovered Women. Pathogens 10, 705.

https://doi.org/10.3390/pathogens10060705

Boehm, E., Kronig, I., Neher, R.A., Eckerle, I., Vetter, P., Kaiser, L. et al., 2021. Novel SARS-CoV-2 variants: the pandemics within the pandemic. Clin. Microbiol. Infect. Off. Publ. Eur. Soc. Clin. Microbiol. Infect. Dis., S1198-743X(21)00262-7. doi:10.1016/j.cmi.2021.05.022.

De Natale, G., Ricciardi, V., De Luca, G., De Natale, D., Di Meglio, G., Ferragamo, A., Marchitelli, V., Piccolo, A., Scala, A., Somma, R., Spina, E., Troise, C., 2020a. The COVID-19 Infection in Italy: A Statistical Study of an Abnormally Severe Disease. J. Clin. Med. 9, 1564. https://doi.org/10.3390/jcm9051564

De Natale, G., De Natale, L., Troise, C., Marchitelli, V., Coviello, A., Holmberg, K.G., Somma, R., 2020b. The Evolution of Covid-19 in Italy after the Spring of 2020: An Unpredicted Summer Respite Followed by a Second Wave. Int. J. Environ. Res. Public Health 17, 8708. https://doi.org/10.3390/ijerph17238708

ECDC, 2021. Threat Assessment Brief: Emergence of SARS-CoV-2 B.1.617 variants in India and situation in the EU/EEA (2021). Eur. Cent. Dis. Prev. Control. Available at:

https://www.ecdc.europa.eu/en/publications-data/threat-assessment-emergence-sars-cov-2-b1617variants [Accessed August 12, 2021].

Liang, T., Cheng., M., Teng, F. et al., 2021. Proteome-wide epitope mapping identifies a resource of antibodies for SARS-CoV-2 detection and neutralization. Sig Transduct Target Ther 6, 166. https://doi.org/10.1038/s41392-021-00573-9

Mattiuzzi, C., Henry, B.M., and Lippi, G., 2021. Is diffusion of SARS-CoV-2 variants of concern associated with different symptoms? J. Infect. doi:10.1016/j.jinf.2021.07.008.

Mourier, T., Shuaib, M., Hala, S., Mfarrej, S,. Alofi, F., Naeem, R., Alsomali, A., Jorgensen, D., Kumar Subudhi, A., Ben Rached, F., Guan, Q., Salunke, R.P., Ooi, A., Esau, L., Douvropoulou, O., Nugmanova, R., Perumal, S., Zhang, H., Rajan, I., Al-Omari, A., Salih, S., Ahamsan, A., Al Mutair, A.A., Taha, J., Alahmadi, A., Khotani, N., Alhamss, A., Mahmoud, A., Alquthami, K., Dageeg, A., Khogeer, A., Hashem, A.M., Moraga, P., Volz, E., Almontashiri, N., Pain, A., 2021. Saudi Arabian SARS-CoV-2 genomes implicate a mutant Nucleocapsid protein in modulating host interactions and increased viral load in COVID-19 patients. medRxiv, doi: https://doi.org/10.1101/2021.05.06.21256706 
Nicastro, F., Sironi, G., Antonello, E. et al., 2021. Solar UV-B/A radiation is highly effective in inactivating SARS-CoV2. Sci Rep 11, 14805. https://doi.org/10.1038/s41598-021-94417-9

Rahman, M.S., Islam, M.R., Alam, A., Islam, I., Hoque, M.N., Akter, S. et al., 2021. Evolutionary dynamics of SARS-CoV-2 nucleocapsid protein and its consequences. J. Med. Virol. 93, 2177-2195. doi:10.1002/jmv.26626.

Shang J, Wan Y, Lou C, Ye G, Geng Q, Auerbach A, Fang L. (2020). Cell entry mechanisms of SARS-CoV-2". Proceedings of the National Academy of Sciences. 117 (21): 11727-11734.

doi:10.1073/pnas.2003138117. PMC 7260975. PMID 32376634.

Starr, T.N., Greaney, A.J., Dingens, A.S., Bloom, J.D., 2021."Complete map of SARS-CoV-2 RBD mutations that escape the monoclonal antibody LY-CoV555 and its cocktail with LY-CoV016". Cell. Reports Medicine, 2 (4): 100255. doi:10.1016/j.xcrm.2021.100255. PMC 8020059. PMID 33842902.

Wu, H., Xing, N., Meng, K., Fu, B., Xue,W., Dong, P., Xiao, Y., Liu, G., Luo, H., Zhu, W., Lin, X., Meng, G., Zhu, Z., 2021. Nucleocapsid mutation R203K/G204R increases the infectivity, fitness and virulence of SARS-CoV bioRxiv 2021.05.24.445386; doi: https://doi.org/10.1101/2021.05.24.445386

\section{Figures}

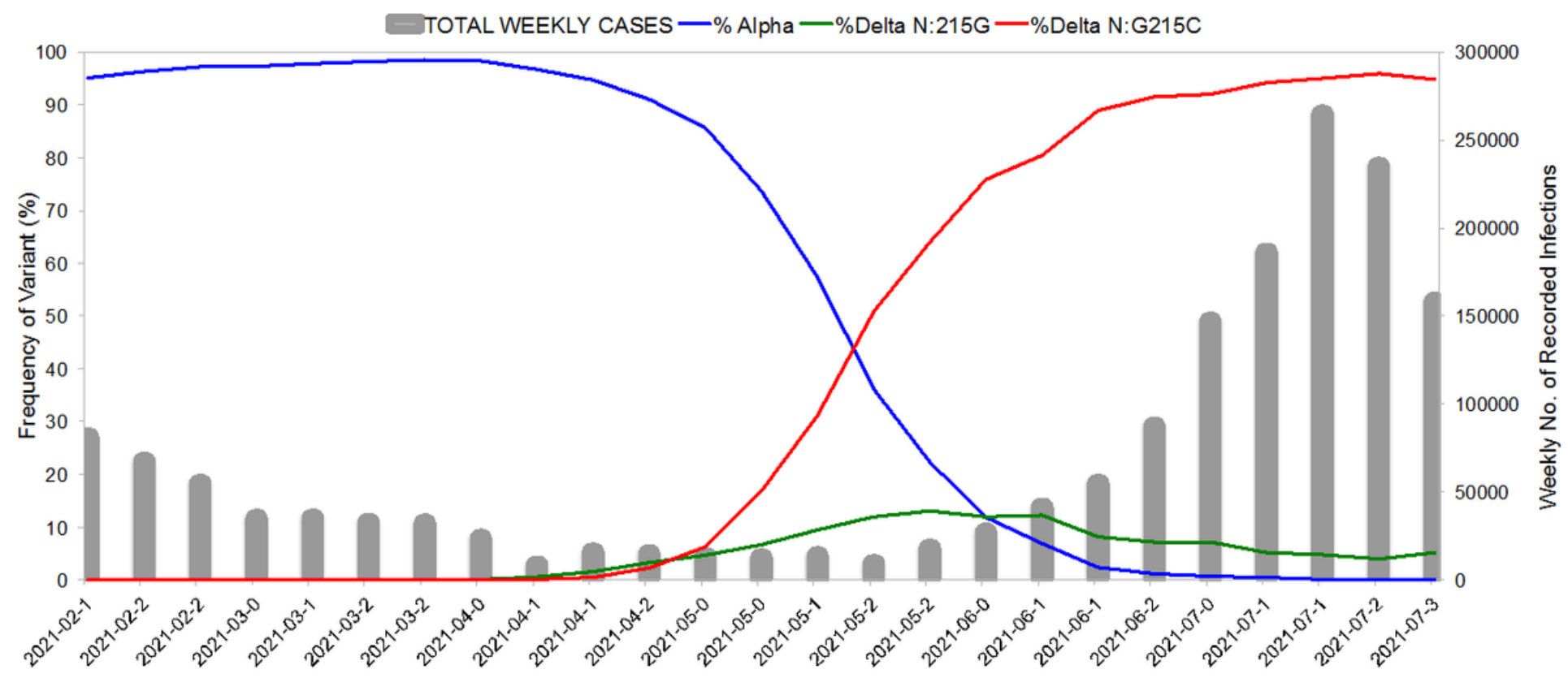

\section{Figure 1}

Time evolution of Alpha, Delta N:215G (WT) and Delta N:G215C variants, from the UK genome catalogue. 


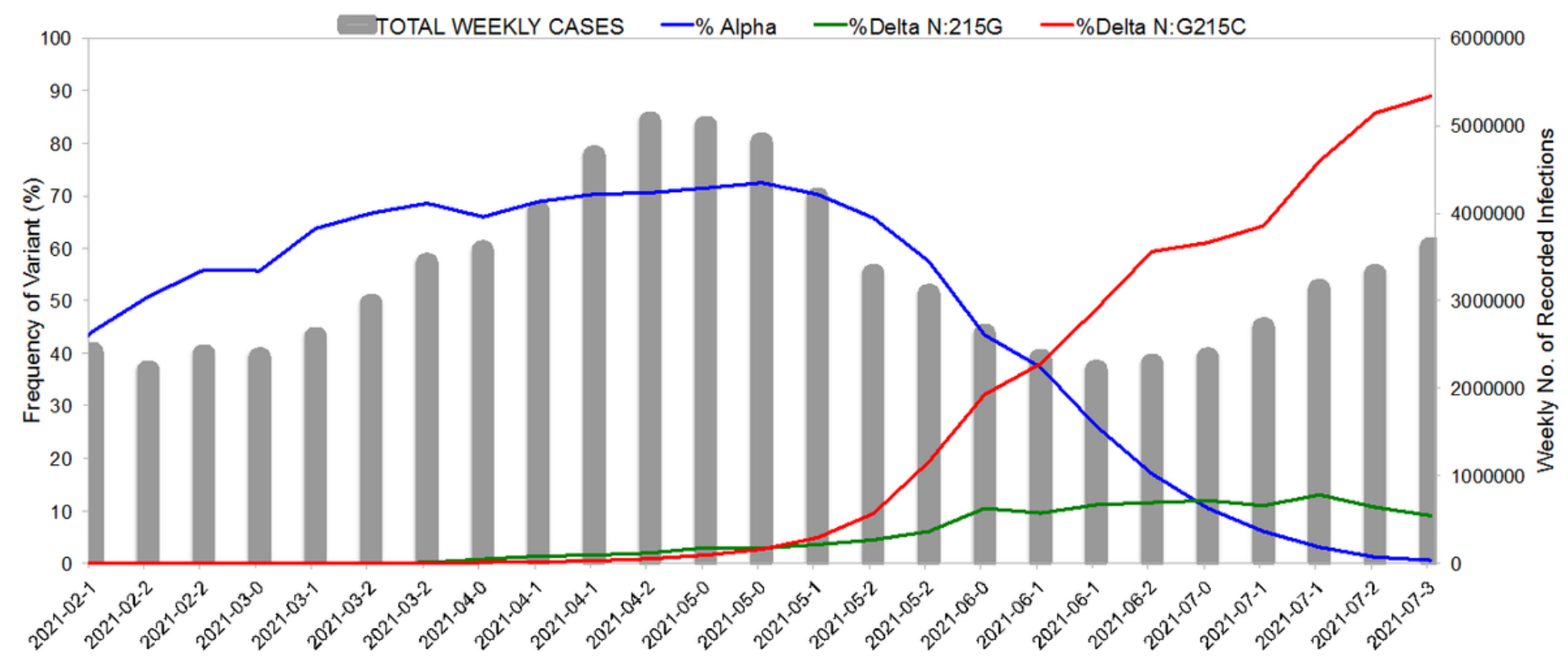

Figure 2

Time evolution of Alpha, Delta N:215G (WT) and Delta N:G215C variants, from the worldwide genome catalogue

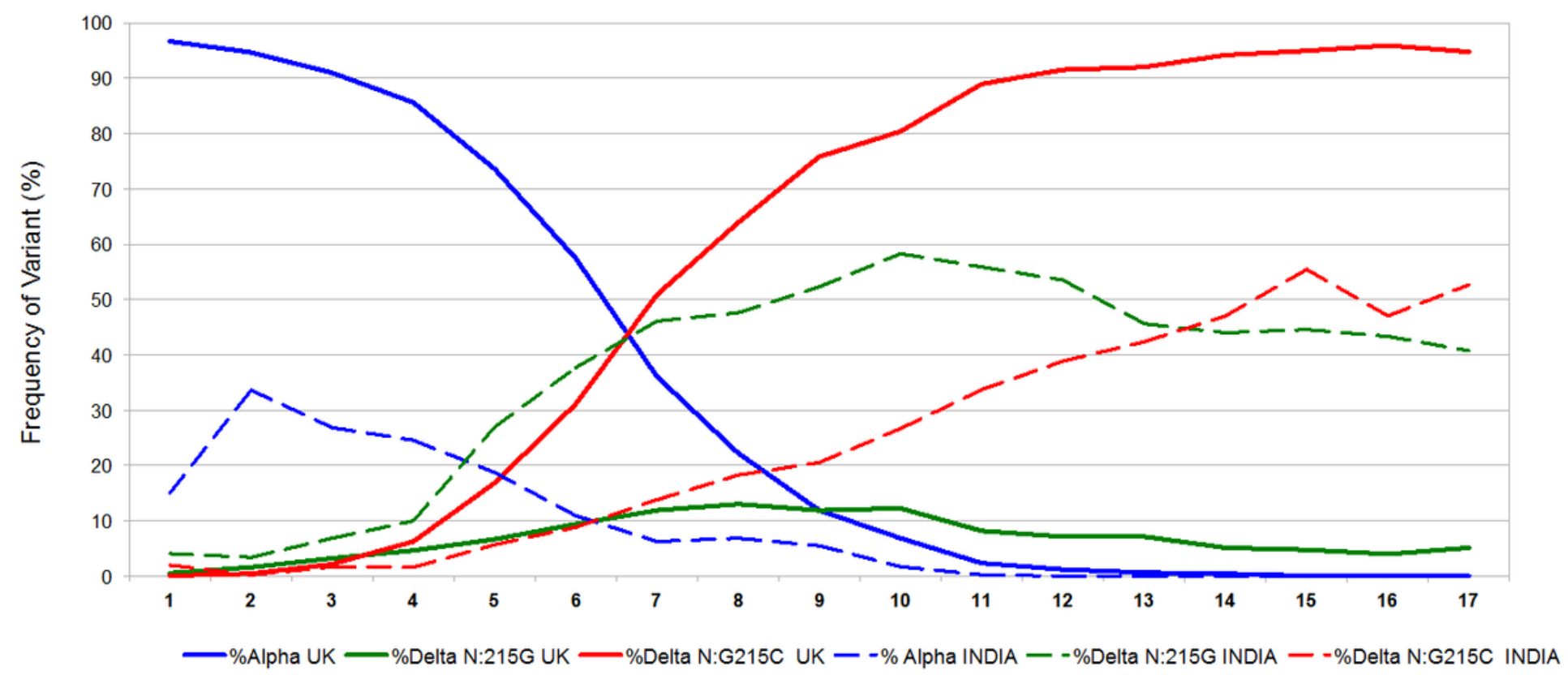

\section{Figure 3}

Comparison of Alpha and Delta variants in the genomes analysed in UK and India. The time scale shows the weeks from an initial time which is 2021-04-10 for UK, whereas the India initial time is shifted so that the rise of Delta variant is roughly the same. The UK times end on 2021 July 31; at the same data, in 
India, the variant Delta N:G215C has reached about $65 \%$ of prevalence, the remaining $35 \%$ being occupied by the Delta variant without the N:G215C mutation. 